Théologiques

Théologiques

\title{
La foi chrétienne dans la société urbaine d'aujourd'hui
}

\section{Norbert Greinacher}

Volume 3, numéro 1, mars 1995

Symbolique urbaine et foi chrétienne

URI : https://id.erudit.org/iderudit/602412ar

DOI : https://doi.org/10.7202/602412ar

Aller au sommaire du numéro

\section{Éditeur(s)}

Faculté de théologie de l'Université de Montréal

\section{ISSN}

1188-7109 (imprimé)

1492-1413 (numérique)

Découvrir la revue

\section{Citer cet article}

Greinacher, N. (1995). La foi chrétienne dans la société urbaine d'aujourd'hui. Théologiques, 3(1), 13-25. https://doi.org/10.7202/602412ar

\section{Résumé de l'article}

Depuis les époques les plus anciennes de l'histoire, et même de la préhistoire, les êtres humains se sont regroupés dans des villes et y ont accordé une place centrale et privilégiée à leurs divinités. Le christianisme s'est lui aussi développé principalement en milieu urbain. Pourtant, l'observation des expériences d'Églises des derniers siècles révèle une distance profonde entre la vie sociale urbaine et les pratiques pastorales modernes. Les Églises d'occident ont failli à leur tâche d'être au coeur des enjeux sociaux urbains contemporains. La vitalité des communautés d'Amérique latine pourrait cependant donner un nouveau souffle à cette mission fondamentale de la foi chrétienne qui consiste à participer, sur le terrain, à la vie même de la cité. 


\title{
La foi chrétienne dans la société urbaine d'aujourd'hui*
}

\author{
Norbert GREINACHER \\ Faculté de théologie catholique \\ Université de Tübingen
}

\section{RÉSUMÉ}

Depuis les époques les plus anciennes de l'histoire, et même de la préhistoire, les êtres humains se sont regroupés dans des villes et y ont accordé une place centrale et privilégiée à leurs divinités. Le christianisme s'est lui aussi développé principalement en milieu urbain. Pourtant, l'observation des expériences d'Églises des derniers siècles révèle une distance profonde entre la vie sociale urbaine et les pratiques pastorales modernes. Les Églises d'occident ont failli à leur tâche d'être au cour des enjeux sociaux urbains contemporains. La vitalité des communautés d'Amérique latine pourrait cependant donner un nouveau souffle à cette mission fondamentale de la foi chrétienne qui consiste à participer, sur le terrain, à la vie même de la cité.

\section{Les trois révolutions dans l'histoire de l'humanité}

En regardant le déroulement de l'histoire de l'humanité, de son origine jusqu'à nos jours, nous pouvons constater trois transformations lourdes de conséquences pour la vie sociale et économique des humains : la révolution rurale, la révolution urbaine et la révolution industrielle. Ces trois révolutions ont profondément transformé le rapport de l'être humain à la nature. Il est vrai qu'elles ont eu lieu à des endroits différents, dans des cultures différentes et à des moments différents. On peut cependant déceler certaines constantes dans le déroulement de l'histoire de l'humanité.

* Traduit de l'allemand par Monika Thoma-Petit. 
Lorsque sont remplies certaines conditions économiques, sociales et culturelles, l'évolution arrive à un stade où se produit un tournant.

La première révolution débuta à l'époque où les hommes du mésolithique, qui avaient jusqu'alors vécu de la chasse et de la cueillette, devinrent des agriculteurs sédentaires et construisirent des lieux d'habitation permanents. J'appelle ce processus décisif de transformation «la révolution rurale " [dörfliche Revolution]. Cette transformation fut précédée par un événement qui s'avéra d'une importance décisive pour l'être humain : la domestication du feu. Nous trouvons des traces de cette révolution rurale dans les habitations en forme de villages en Mésopotamie autour de 7000 av. J.-C. En Europe centrale et dans la partie ouest de l'Europe, la sédentarisation devint définitive au quatrième et au troisième millénaire av. J.-C., et pour le nord de l'Europe au deuxième millénaire avant notre ère seulement.

"L'humain évolué ... est un animal qui construit des villes 1 ", a écrit Oswald Spengler. Il fait ainsi référence à la deuxième révolution, à savoir l'émergence de civilisations urbaines. Cette révolution eut lieu au cinquième millénaire av. J.-C. Elle consista essentiellement dans le fait que la société avait subi des transformations structurelles profondes, dues à la division du travail, et qu'elle se distinguait ainsi de la société paysanne. Ces transformations socio-économiques créèrent les conditions qui rendirent possible l'émergence d'une civilisation. Le résultat de cette révolution est encore aujourd'hui le fondement de notre existence humaine.

Le troisième et plus important tournant dans l'histoire de l'humanité fut la révolution industrielle. Hans Freyer l'a décrite de la manière suivante :

On peut difficilement nier qu'au tournant du XIXe siècle a débuté une ère de l'histoire humaine d'importance majeure, comparable seulement, peut-être, à la sédentarisation de l'humain au début de l'époque néolithique. Construire des machines, les faire travailler à sa place et se rendre dépendant, jusque dans son âme, de leur fonctionnement cette décision a autant transformé l'existence humaine que la décision d'antan d'élire domicile entre quatre poteaux et de lier son destin à un morceau de terre qu'on cultive comme un jardin 2 .

1 Oswald SPENGLER, Der Untergang des Abendlandes II. München, Beck, 1922, p. 106.

2 Hans FREYER, Theorie des gegenwärtigen Zeitalters. Stuttgart, Deutsch Verlags Anstalt, 1956 ( 2 éd.) p. 81. 


\section{La ville comme domicile de Dieu}

La religion eut une fonction très importante lors de l'émergence des villes. Puisque, de la procréation jusqu'à la mort, la vie humaine était alors accompagnée de significations religieuses, la religion avait pour l'ensemble de la vie humaine une si grande importance qu'il était impensable de ne pas réserver aux dieux un domicile au sein des habitations. L'historien de la culture Lewis Mumford décrit ce phénomène de la façon suivante : "La cité ancienne exprimait dans tous ses éléments la foi que l'humain n'a été créé à nulle autre fin que d'être au service et à la gloire de son dieu. Et ceci était le véritable fondement de l'existence de la cité $^{3}$. "Semblable à la façon dont le sanctuaire était devenu le point central de l'habitation villageoise, le temple a joué un rôle important dans l'émergence des villes et dans la vie sociale de la cité.

Le temple était étroitement lié, par son organisation spatiale, au palais du roi, qui était souvent un prêtre-roi. "La cohésion spirituelle des citadins étair assurée par une autorité centrale représentée par le temple et ses prêtres, ou par la cour du souverain, dont les attributs étaient au moins ceux d'un demi-dieu ${ }^{4}$."

Le domicile des dieux était la cité. C'est là qu'ils étaient vénérés, c'est là qu'on leur érigeait des sanctuaires. Dans la plupart des cas, la fondation des villes était justifiée par la volonté divine. La ville eut sa légitimité par la volonté des dieux. Nous citons à nouveau Lewis Mumford:

La ville est tout d'abord la création d'un roi ... qui agit au nom d'une divinité. Le premier geste du roi, l'origine véritable de son autorité et de son pouvoir, est l'érection d'un temple au sein d'un domaine sacré entouré de puissantes fortifications. La construction d'une deuxième muraille dont la fonction était la protection de toute la communauté qui était au service de cette divinité, transforme l'ensemble de cet espace en un lieu saint : la ville ${ }^{5}$.

À l'origine, la ville était donc le lieu de résidence d'un dieu et avait un caractère sacré.

3 Lewis Mumford, La cité à travers l'histoire. Paris, Seuil, 1964, p. 92.

4 Robert M. FISCHER, The Metropolis in Modern Life. New York, Doubleday, 1955, p. 8 .

5 Lewis MUmFord, "Utopie, Stadt und Maschine" dans F. E. MANUAL, Wunschtraum und Experiment. Vom Nutzen und Nachteil utopischen Denkens. Freiburg, Herder, 1970, p. 40. 


\section{La foi juive entre Babylone et Jérusalem}

Le rapport à la ville qu'entretenaient les membres du peuple d'Israël est profondément ambigu. D'une part, nous lisons des commentaires extrêmement critiques concernant des villes comme Sodome et Gomorrhe, ainsi que la critique de la ville chez les prophètes Amos, Michée et Isaïe. Et ce n'est probablement pas un hasard si Caïn, le fratricide, fut le premier constructeur de ville ( $\mathrm{G} n 4,17$ ).

D'autre part, nous lisons chez Jétémie la description suivante de la ville de Babylone, lieu de la "captivité babylonienne " et appelée plus tard dans le Nouveau Testament Babylone la Prostituée ( Ap 17,1-8):

Voici le texte de la lettre que le prophète Jérémie expédia de Jérusalem aux anciens en captivité, aux prêtres et aux prophètes et à tout le peuple que Nabuchodonosor avait déportés de Jérusalem à Babylone... Ainsi parle Yahvé Sabaot, le Dieu d'Israël, à tous les captifs déportés de Jérusalem à Babylone. Bâtissez des maisons et installez-vous; plantez des jardins et mangez leurs fruits; prenez femme et procréez des fils et des filles; choisissez des épouses pour vos fils, donnez vos filles à des hommes et qu'elles enfantent des fils et des filles; multipliez là-bas : ne diminuez pas! Recherchez le bien de la ville où je vous ai déportés. Priez Yahvé en sa faveur, car de sa prospérité dépend la vôtre. ( Jr 29,1-7)

Ceci amène Heinrich Jacob à la conclusion que la ville se trouve au sein d'une tension entre Babel et Jérusalem. "La ville peut être un symbole autant pour le salut que pour le malheur ${ }^{6}$."

\section{De Galilée à Jérusalem}

"À partir de ce moment, Jésus commença à expliquer à ses disciples qu'il devait monter à Jérusalem" ( $M t$ 16,21). La vie de Jésus est fortement marquée par une concentration sur les villes ${ }^{*}$. Il se trouve toujours en chemin entre deux villes. Sa vie errante débute lorsqu'il se détache du village de Nazareth. Malgré cela, il dénonce publiquement des inconvénients de la ville, par exemple les pratiques de commerce au temple ( $M t$ 24,1s.), et il subit lui-même dans la ville de Jérusalem, et par les autorités de la ville, l'échec public de sa prédication, par sa mort

6 Heinrich JACOB, “Seelsorge in einer Grossstadt », Lebendige Seelsorge 42 (1992), p. 14.

* N.D.L.R. Pour un avis différent, voir plus loin l'article d'André Myre. 
sur la croix. Jésus réfléchit sur les difficultés de la vie urbaine, par exemple à l'occasion du débat sur l'impôt du temple $(M t$ 17,22) et lorsqu'il se révolte contre la loi du Sabbat ( Mc 1,29-31). Il critique aussi de façon virulente les villes impénitentes ( Mt 11,20-24).

Jésus se trouve ici dans la tradition de Jean le Baptiste qui est le critique de la ville par excellence, car il prêche la conversion dans le désert. Mais le mouvement migratoire [Wanderbewegung] de Jésus est, à l'encontre de celui du Baptiste, orienté vers Jérusalem, la ville où se réalisera le royaume de Dieu $^{7}$.

\section{Le christianisme et l'urbanisation}

Lorsqu'on regarde les premières tentatives de mission du christianisme primitif en Palestine telles que nous les connaissons surtout à partir des Actes des Apôtres, on voit clairement que les premières communautés chrétiennes se sont d'abord constituées dans des zones d'habitation urbaines. Jérusalem demeura d'abord le centre. De là, Philippe partit vers la Samarie ( $A c 8,5)$. Mais plus tard, "il annonça la bonne nouvelle dans toutes les villes qu'il traversait, jusqu'à Césarée » ( $A c$ 8,40). Puis nous entendons parler de communautés chrétiennes à Damas ( $A c 9)$, Lydda ( Ac 9,32), Joppé ( $A c$ 9,36), Césarée $(A c 10)$ et Antioche ( $A c 11$ ).

Le christianisme primitif fut d'abord presque exclusivement une religion des villes. Les voyages de mission de Paul l'amenèrent d'abord dans des villes; lors de son premier voyage il alla à Salamine ( $A c$ 13,5), Paphos ( Ac 13,6-12), Pergé ( Ac 13,13), Antioche de Pisidie (Ac 13,1450 ), Iconium ( $A c$ 13,51-14,5), Lystres (Ac 14,6), Derbé ( Ac 14,6); et lors du deuxième voyage, à Troas ( $A c$ 16,8-10) Philippes ( $A c$ 16,12-40), Thessalonique ( $A c$ 17,1-9), Bérée ( $A c$ 17,10-14), Athènes ( $A c$ 17,15-34), Corinthe ( $A c$ 18,1-16), Éphèse ( $A c 18,19-21$ ), et à la fin de ses voyages enfin à Rome ( $A c$ 28,14).

L'Apocalypse nous apprend qu'il y avait, au temps des apôtres, d'autres communautés chrétiennes à Smyrne (2,8), Pergame $(2,12)$, Thyatire (2,18), Sardes (3,1), Philadelphie (3,7) et à Laodicée $(3,14)$. L'épître aux Colossiens témoigne d'une communauté à Colosses.

Comme le montre la fondation des communautés par Paul, le christianisme primitif se propagea surtout en suivant les grands axes de com-

7 Cf. Friedhelm BorgGrefe, Kirche für die Grossstadt. Heidelberg, Quelle und Meyer, 1973, p. 42 s. 
merce et de communication de ce temps. J. Chelini écrit : "Le christianisme primitif était presque exclusivement un phénomène urbain... L'évolution du christianisme reste étroitement liée aux villes. Son destin leur semble lié d'une manière toute particulière. Partout où les villes sont nombreuses, nous trouvons l'existence du christianisme ${ }^{8}$." Ni les Actes des Apôtres, ni les Épîtres ne nous donnent des indications explicites quant à l'existence d'une communauté en milieu rural. Il n'est pas exclu que le message du Christ pénétra en milieu rural et qu'il y ait eu quelques chrétiens. Mais le centre de gravité se trouve nettement dans les villes. À l'ouest surtout, on peut parler d'une diffusion presque exclusivement urbaine. W. Croce écrit : "Lorsque les apôtres laissèrent derrière eux, dans les communautés chrétiennes nouvellement fondées, des responsables pour diriger l'Église - comme Timothée à Éphèse ou Tite à Crète - il s'agissait alors d'évêques, et leurs villes avec les régions alentours constituèrent les premiers diocèses ${ }^{9}$. "

La répartition des sièges épiscopaux à l'époque post-apostolique nous montre également que le siège d'un évêque était toujours lié à une ville. C'est seulement vers la fin du troisième et au début du quatrième siècle qu'on trouve des paroisses rurales au sens où l'on chargea un prêtre de la pastorale auprès des chrétiens qui vivaient dans des villages situés loin des villes. Le christianisme demeura donc longtemps, à l'époque postapostolique, un phénomène essentiellement urbain. "Pas d'évêque sans ville, pas de communauté chrétienne sans évêque... Le siège épiscopal est celui d'une ville, jamais d'une région; on est évêque d'un lieu, non d'une province ou d'un district administratif quelconque ${ }^{10}$. "

Il est symptomatique que le christianisme primitif désigne le gentil simplement par le mot "paganus ", c'est-à-dire celui qui reste à la campagne, le campagnard. Ce fait déjà nous permet de voir combien le christianisme primitif était lié à la ville. Max Weber a raison d'écrire :

C'est un fait que la religiosité chrétienne primitive est une religiosité urbaine; comme l'a démontré Harnack de façon convainquante, toutes choses étant égales par ailleurs, l'importance du christianisme augmente en fonction de l'importance de la ville... Il est tout à fait invraisemblable qu'une religiosité communautaire organisée comme le

8 J. ChelINI, La ville et l'Église. Paris, Cerf, 1958, p. 122s.

9 W. CROCE, « Die Geschichte der Pfarre» dans Hugo Rahner (éd), Die Pfarre. Freiburg, Herder, 1956, p. 15.

10 J. CHELINI, La ville et l'Église, p. 124s. 
devint celle du christianisme ait pu se développer en dehors d'une vie sociale urbaine, au sens occidental du terme urbain. Car elle présupposait comme concepts déjà existants l'éclatement des frontières érigées en tabou entre les clans, le concept de fonction, la compréhension de la communauté comme institution, comme forme corporative au service de buts pratiques ( sachlich). Elle a contribué à renforcer ces conceptions, dont la reprise fut facilitée par le développement des villes dans l'Europe médiévale ${ }^{11 .}$

L'Église a par ailleurs sans aucun doute influencé de façon décisive la vie urbaine au moyen âge, et l'Église médiévale fut, à son tour, marquée par la ville.

Dans ce contexte, les monastères ont aussi joué un rôle important. Ils ont eu une grande influence sur la vie urbaine et furent même souvent à l'origine d'une nouvelle ville. Lewis Mumford a raison d'écrire: "Les abbayes de Westminster, Clairvaux, Saint-Denis, Monte Cassino et Fulda dominent la vie urbaine jusque dans ses formes architecturales, et dans une mesure qui n'avait rien à voir avec leur nombre ${ }^{12}$. »

Alors que la vie urbaine médiévale détermina et marqua dans une large mesure la structure organisationnelle de l'Église et que le style de vie urbain devint largement déterminant pour la vie de l'Église dans son ensemble, la ville fut à son tour marquée par l'Église. L'ensemble de la vie sociale des gens dans ces villes fut profondément imprégné de représentations chrétiennes et déterminé par la vie de l'Église. L'Église et la société urbaine ne formaient plus qu'une seule entité, tout comme l'Église et l'Empire. Max Weber exprime ce phénomène de la façon suivante :

La ville médiévale était un ensemble cultuel. L'Église de la ville, le saint patron de la ville, la participation des citoyens à l'eucharistie, les fêtes religieuses officielles de la ville, tout cela allait de soi. Mais le christianisme avait enlevé au clan toute signification rituelle. Les communautés chrétiennes étaient au plus intime d'elles-mêmes une association confessionnelle d'individus croyants et non pas une association rituelle de clans ${ }^{13}$.

11 Max WEBER, Économie et société. Paris, Plon, 1971, p. 494.

12 Lewis MUMFORD, La cité, p. 296.

13 Max WEBER, Wirtschaft und Gesellschaft II. Köln, 1964, p. 949. 


\section{Le désenchantement de la ville}

Dans le contexte global de nos réflexions, nous devons maintenant considérer un phénomène qui, bien que n'étant pas une réalité urbaine proprement dite mais plutôt un processus de la société en général, a été articulé de façon particulière dans la société urbaine et fut d'une grande importance pour le destin du christianisme dans cette société. Albert Mirgeler décrit ce processus de la façon suivante:

Ce qui fut décisif pour toute la suite de l'histoire, ce fut le fait que, au plus fort de la querelle des investitures (i.e. à la fin du XI et au début du XIIe siècle ), la jeune science européenne élabora la distinction entre les concepts de spirituel [das Geistliche] et de temporel [das Weltliche] ... Lorsque Grégoire VII tira les conclusions de cette tradition et contesta que l'empereur puisse avoir des droits de disposition dans l'Église, l'union entre l'Empire et l'Ecclesia, datant de l'époque de Constantin, éclata dans ses fondements. ... L'empire occidental, surélevé, de surcroît, avec Otton le Grand par l'idée d'un ministère de grand prêtre vétéro-testamentaire, fut désacralisé de façon radicale et l'empereur fut laïcisé ${ }^{14}$.

L'évolution subséquente eut cependant ceci de particulier que cette distinction fondamentale entre le spirituel et le temporel qui apparaît même textuellement dans le concordat de Worms en 1122, n'a pas eu d'effet tout au long du moyen âge jusqu'aux temps modernes, c'est-à-dire qu'elle n'a eu aucune pertinence sociale. L'union de fait entre le trône et l'autel fut conservée, sous différentes formes de compromis, jusqu'à la révolution française de 1789 et la révolution russe de 1917. La distinction de principe entre le spirituel et le temporel eut toutefois comme conséquence que cette union constitua une synthèse culturelle pleine de contradictions internes, perpétuellement menacée de conflits.

Nous nous trouvons donc devant le fait remarquable que les racines du processus de sécularisation plongent jusqu'au début du moyen âge, mais que cela a pris des siècles avant qu'il évolue suffisamment pour devenir porteur d'histoire.

Max Weber a choisi l'image du "désenchantement " [Entzauberung] pour décrire ce processus, dans la mesure où le cosmos entier et la nature ne sont plus considérés comme symboles de la puissance divine, comme

14 Albert Mirgeler, Rückblick auf das abendländische Christentum. Mainz, Matthias Grunewald Verlag, 1961, pp. 109-114. 
"vestigia dei ", mais comme réalités du monde, dont l'homme peut connaître les lois et dont il peut disposer. Max Weber écrit ceci :

Plus l'intellectualisme fait reculer la foi en la magie et désenchante ainsi les processus du monde qui perdent leur sens magique et " existent" ou "se déroulent" seulement, mais qui ne "signifient" plus rien, et plus il devient urgent d'exiger du monde et de la "conduite de la vie" considérés dans leur ensemble qu'ils soient ordonnés de façon significative et qu'ils revêtent un sens 15 .

Tout ce processus complexe doit être vu en lien direct avec la ville; ceci découle de notre thèse que l'urbanisation constitue pour ainsi dire la pointe de l'évolution sociale dans son ensemble. On comprend donc facilement que ce processus de "désenchantement " n'ait pas d'abord débuté parmi la population paysanne qui, à cause de ses liens étroits avec la nature et sa mentalité plutôt persévérante, tend davantage à résister au désenchantement du monde que les gens dans la ville, là où sont d'abord apparues et ont dû être réglées les tensions entre les institutions ecclésiales et séculières.

\section{La ville aujourd'hui : Moloch et lieu de liberté}

L'urbanisation est un processus mondial qui a débuté au siècle dernier, même si le développement des grandes villes est essentiellement un phénomène du XXe siècle. Vers $1900,90 \%$ de la population mondiale vivait encore à la campagne. En 1985, 2,7 milliards de personnes vivaient à la campagne, 2,1 milliards habitaient les villes. L'an 2000 sera le tournant. Selon les estimations des Nations Unies, 2,9 milliards de personnes vivront alors à la campagne et 3,1 milliards en ville. Pour l'an 2025, les estimations des Nations Unies prédisent que 2,8 milliards de personnes habiteront la campagne alors que 5,3 milliards vivront en ville ${ }^{16}$.

Le développement des villes de l'Amérique du Sud, d'Asie et d'Afrique connaît déjà cette accélération et, depuis longtemps, la plupart de ces villes ne sont plus capables de faire face aux problèmes reliés à l'approvisionnement en énergie, aux besoins de logements et de santé publique, au chômage, à la pollution de l'environnement et aux écarts entre les couches sociales. D'un côté, la ville est devenue un "Moloch *. Les villes nord-américaines ne sont pas les seules où un individu ne peut plus oser se promener seul la nuit ou le soir venu. Il est confronté à d'im-

Max WEBER, Wirtschaft und Gesellschaft, II, p. 949.

Süddeutsche Zeitung, 13 février 1986. 
portants risques de sécurité, compte tenu de la violence, des vols à main armée et des menaces pour la vie même, qui existent dans la ville. Seul les membres des couches sociales aisées peuvent se payer une protection sous forme d'un service de police privée.

D'un autre côté, la ville est demeurée un lieu de liberté individuelle. Nulle part ailleurs qu'en ville est-il possible, pour le citoyen ou la citoyenne, de décider avec autant de liberté de sa propre manière de vivre. Le processus postmoderne de l'individualisation est localisé avant tout dans la société urbaine. Ulrich Beck et Elisabeth Beck-Gernsheim l'ont décrit de la façon suivante :

Développement du soi dans ses blocages et ses contraintes sociales tout autant que des possibilités de les assouplir ou de les faire éclater, par son action pratique, et ensuite d'avoir un impact ( au-delà du mariage, de la famille et des rapports entre les sexes) sur le travail, la politique et les institutions, les rapports avec la nature et la technologie - toujours en lien avec le problème central : découverte et épanouissement du soi dans le social et définition d'un social qui permet la libération et l'épanouissement réciproque ${ }^{17}$.

\section{L'Église dans la ville d'aujourd'hui*}

Dès le début, les deux grandes Églises ont, à quelques exceptions près, refusé de prendre acte de la réalité de la société urbaine, du processus d'industrialisation et de sécularisation, et de développer une pastorale moderne pour le milieu urbain.

Il est vrai qu'au XIX ${ }^{e}$ siècle, on a construit dans les villes un grand nombre de nouvelles Églises pour les deux confessions, afin de fournir des services aux nouveaux arrivants et de continuer à manifester la nécessité de la présence de l'Église. Mais les tâches des communautés chrétiennes consistèrent surtout en l'organisation d'activités d'assistance publique pour des individus pauvres afin de pallier aux insuffisances des services municipaux. Les Églises fonctionnaient comme des institutions qui existaient à côté d'autres domaines d'importance sociale, par exemple les associations. "Dans les villes, les processus nécessaires de socialisation n'ont pas lieu dans les Églises, mais dans les associations; celles-là pous-

17 Ulirich BECK et Elisabeth BECK-GERNSHEIM, Das ganz normale Chaos der Liebe. Frankfurt, Suhrkamp, 1990, p. 64.

* N.D.L.R. On comprendra que les pages suivantes réfèrent au contexte allemand de l'aureur. 
sent comme des champignons, et favorisent le développement du sens communautaire et des traditions nationales, culturelles et musicales ${ }^{18}$."

Les Églises n'ont joué aucun rôle dans d'autres problèmes massifs causés par l'urbanisation, par exemple dans la problématique du logement ou de l'environnement, et surtout en ce qui concerne les effets de l'industrialisation, par exemple les problèmes de la sécurité de l'emploi ou des rentes, la protection des travailleurs licenciés, l'assurance-santé, etc. Bien que l'humanisation du processus du travail aurait sûrement dû être une des tâches des Églises, il n'y a eu en ce sens que quelques rares initiatives, par exemple celles de Mgr von Ketteler, d'Adolf Kolping et de Johann Hinrich Wichern. La communauté ecclésiale qui, pour les gens nouvellement arrivés en ville, peut représenter d'abord un lieu d'appartenance, leur apparaît bientôt si éloignée de leur réalité qu'ils s'organisent dans d'autres institutions qui se préoccupent davantage de leur réalité, par exemple les partis socialistes et les syndicats. Il est vrai que les Églises se dévouent à des tâches sociales et font aussi du travail de formation; mais la plupart du temps, ces initiatives sont orientées vers les individus et les familles, et non vers les structures. Les Églises considèrent que la mise en place d'une aide sociale individuelle est de leur ressort mais, tout compte fait, elles ne participent pas à la vie sociale publique.

Cette attitude fondamentale des Églises face à la vie dans les grandes villes au $\mathrm{XIX}^{\mathrm{e}}$ et dans la première moitié du $\mathrm{XX}^{\mathrm{e}}$ siècle continue à perdurer jusqu'à aujourd'hui et a contribué à accélérer la sécularisation de la société urbaine. "L'Église a failli jusqu'à maintenant à la tâche de s'approprier d'une façon fructueuse sa propre urbanité ${ }^{19}$. "Ceci s'explique essentiellement par le fait que les Églises ont affronté la ville à partir de leurs concepts ruraux. Cette fausse approche pèse aujourd'hui d'autant plus lourd qu'on doit constater un mode de vie urbain partout dans la société dans son ensemble. "Ce qui se passe aujourd'hui dans les villes, se trouvera demain à la campagne ${ }^{20}$."

18 Friedrich BORGGREFE, Kirche für die Grossstadt, p. 63.

19 Hans Joachim HöN, « Religion in der City - oder: Den Glauben zu Markte tragen? », Lebendige Seelsorge 43 (1992), p. 14

20 Ibidem. 


\section{Le mouvement des communautés de base en Amérique Latine}

Les villes explosent avant tout dans les pays du "tiers-monde". Ceci est particulièrement vrai pour l'Amérique Latine, le continent qui est, dans le tiers-monde, le plus fortement urbanisé. Les deux tiers de la population vivent dans des villes ou autour des villes dans des quartiers très éloignés de la city. À $\mathrm{La} \mathrm{Paz}$, par exemple, tout autour de la city, située au fond de la vallée, les gens se sont installés jusque haut sur les pentes des montagnes. La plupart des nouveaux arrivants s'installent dans de telles zones de misère qui ceinturent les villes. Les barrios, favelas, pueblos jovenes manquent de toutes les infrastructures minimales. Les habitants doivent chercher leur eau très loin, le manque de canalisations augmente les risques d'épidémies. La société en marge de la ville est marquée par le déracinement, le manque d'emplois, des familles nombreuses et une pluralité colorée d'ethnies différentes; elle est souvent menacée par des travaux d'évacuation de la part des autorités, puisque les règlements municipaux interdisent souvent, comme à Lima par exemple, l'installation d'abris de fortune en bordure de la ville. Malgré tout cela, la plupart des gens sont marqués par une religiosité populaire catholique qui constitue la base de la formation de communautés organisées de façon autonome par leurs membres. "Ici émerge dans les villes, une nouvelle réalité ecclésiale pour laquelle il y a une combinaison de motivations religieuses et sociales $^{21}$. " Ici, on réussit, à partir de la situation dans la ville et en faisant face au combat quotidien pour la survie, à créer une forme vivante de communauté ecclésiale qui ne passe pas à côté de la réalité des gens, qui est contextuelle et qui ne vient pas d'en haut, mais qui est en revanche favorisée par la conférence des évêques de l'Amérique Latine ${ }^{22}$.

\section{Perspectives d'avenir}

L'exemple des communautés de base en Amérique latine nous apprend que l'Église dans la ville est une activité de la communauté ecclésiale dans son ensemble, et qu'elle ne dépend pas des seules activi-

21 Michael SEVERNICH, "Formiert sich eine Stadt-Kirche? », Lebendige Seelsorge 43 (1992), pp 6-13, ici p. 9.

22 Die Kirche Lateinamerikas - Documentation der II. und III. Generalversammlung des lateinamerikanischen Episkopates in Medellin und Puebla. Stimmen der Weltkirche Nr. 8. Hg. vom Sekretariar der Deutschen Bischofskonferenz, Bonn, 1985, ici Puebla Nr. 96. 
tés ou initiatives d'un curé. La communauté ecclésiale dans son ensemble se laisse interpeller à la solidarité par les exigences de son milieu.

Les conditions de la société urbaine exigent une forme de pastorale différente de celle pratiquée pendant longtemps selon le modèle classique de la pastorale de village. Les nouveaux champs d'action d'une communauté chrétienne en milieu urbain sont, par exemple, des gestes de solidarité envers des concitoyennes et concitoyens étrangers, des personnes en attente d'un statut de réfugié, des personnes frappées par le chômage, des personnes qui élèvent seules leur famille et des personnes âgées vivant seules. Une communauté protestante de Stuttgart a fondé une Église-hôte (Vesperkirche), c'est-à-dire, qu'elle ouvre les portes de son église, de Noël jusqu'à Pâques, pour y accueillir des personnes sans abri, des chômeurs et d'autres personnes en marge de la société afin de leur offrir un abri, des repas et un lieu d'appartenance. Ceci me semble un exemple authentiquement chrétien.

Il importe donc d'abandonner deux éléments structurels de la pastorale qui s'avèrent de plus en plus dépassés: d'une part la forme sociale de "l'Église populaire", c'est-à-dire l'identité entre la population et l'Église; d'autre part le principe unique de la paroisse territoriale. Les deux correspondent davantage à la situation de l'Église en milieu rural et sont mal adaptés à la société urbaine.

Les Églises devront s'habituer au fait qu'elles ne sont plus, dans la société urbaine, les institutions qui déterminent les normes et peuvent exiger des légitimations. Elles devront sérieusement faire face au processus de "désenchantement ", ce qui signifie qu'elles constitueront dorénavant une minorité dans l'ensemble de la société urbaine. Espérons qu'elles ne le feront pas à la manière d'une secte qui se ferme et crée, à l'aide de ses structures internes, un lieu d'appartenance confortable et autosuffisant, mais plutôt comme une Église consciente de sa situation minoritaire qui, fidèle à la tradition prophétique, élève sa voix contre les injustices sociales multiples de la ville, et qui se solidarise dans sa pratique avec les plus petits et les plus démunis.

... dans la mesure où vous l'avez fair à l'un de ces plus petits de mes frères, c'est à moi que vous l'avez fait. ( Mt 25,40) 